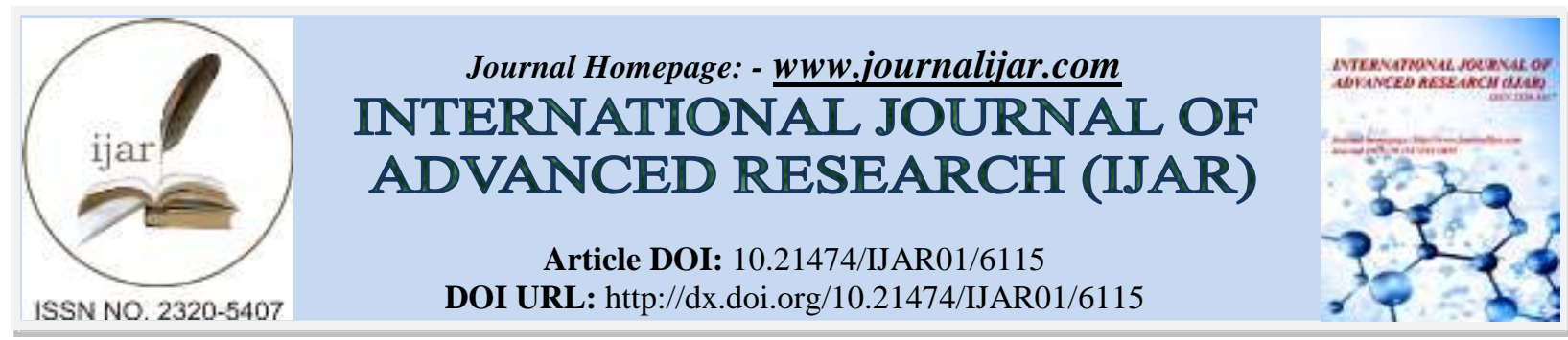

RESEARCH ARTICLE

\title{
CASE REPORT: PSUEDO-THROMBOTIC THROMBOCYTOPENIC PURPRA AND COBALAMINE DEFICIENCY.
}

Housam Almadani, Ziyad T. Mirza and Banan Alsaied.

Department of pediatrics, Hematology Oncology, King Fahd Armed Forces Hospital, Jeddah, Saudi Arabia.

\section{Manuscript Info}

\section{Manuscript History}

Received: 20 October 2017

Final Accepted: 22 November 2017

Published: December 2017

Keywords:-

B12 Deficiency, pseudo-thrombotic thrombocytopenic purpra, TTP, pancytopenia, Schistocyte

\section{Abstract}

Pseudothrombotic microangiopathy is thrombocytopenia, anemia and schistocytosis caused by Cobalamine deficiency. It could be easily misdiagnosed as microangiopathic hemolytic anemia.

Evaluation of lactate dehydrogenase, reticulocyte count, peripheral blood smear, platelet count, cobalamine and homocysteine level are crucial for differentiating pseudothrombotic microangiopathy from a true microangiopathic hemolytic anemia.

A case of pseudothrombotic microangiopathy due to severe vitamin B12 deficiency is presented.

Copy Right, IJAR, 2017,. All rights reserved.

\section{Introduction:-}

Thrombotic Thrombocytopenic Purpura (TTP) is characterized by microangiopathic hemolytic anemia, thrombocytopenia, and neurologic symptoms or renal injury with the disease process driven by a severe deficiency of ADAMTS13, metalloprotease and disintegrin that cleaves Ultra Large Von Willebrand Factor multimers (ULVWF). 123

Vitamin B12 deficiency can present with microangiopathy and can be mistaken for TTP ${ }^{4}$.

Inadequate consumption of animal foods is considered the most common cause of severe vitamin B12 deficiency worldwide in pediatric age group ${ }^{5}$. Most of the Cobalamine deficient cases have only mild haematological findings, in approximately $10 \%$ of patients presents with severe anemia, pancytopenia and hemolytic anemia ${ }^{6}$.

\section{Case report:-}

A 2 years old boy was admitted to hospital with decreased oral intake, decreased appetite, fever and vomiting once. On examination the patient looked pale, apathy, weak; weight and height below $5^{\text {th }}$ centile; and his sclerae were icteric. Laboratory investigations revealed macrocytic anemia (hemoglobin 6.6g/dl; leukopenia (neutrophil1300); and thrombocytopenia (58000). Reticulocyte count was 0.039. Lactate dehydrogenase (LDH) was 3707u/l. Direct Coombs and glucose-6-phosphate dehydrogenase deficiency tests were all negative. Alkaline phosphatase was high 991.7u/l. Plasma homocysteine level was elevated 27.6. A peripheral blood smear showed schistocytes and hypersegmented neutrophils .B12 level was $77.12 \mathrm{pg} / \mathrm{ml}$. folate serum was normal. Vitamin D was low and serum Iron was normal. ADAMTS13 was normal. Bone marrow aspiration study demonstrated megaloblastic erythropoiesis and ruled out leukemia. Intramuscular cyanocobalamin was started and patient respond very well. 


\section{Discussion:-}

Inadequate consumption due to dietary restriction or unavailable access to animal sourced food is the most common cause of Vitamin B12 deficiency in infants and children. ${ }^{6}$

In our patient, he was on exclusive breastfeeding. His four year-old sister presented 8 months later with decreased activity and appetite, and vomiting with evidence of hemolytic anemia and pancytopenia. Her lab consisted with elevated LDH (6310 U/L) and Schistocytes in peripheral blood smear, and low vitamin D.

Cobalamine deficiency is relatively common in the general population, with prevalence estimates ranging from 4$15 \%$, it should be considered in the differential diagnosis of patients with microangiopathic hemolytic anemia. ${ }^{7}$

In vitamin B12 Deficiency pseudothrombotic microangiopathy is related to ineffective erythropoiesis \& intramedullary destruction of erythrocytes. ${ }^{8}$

In addition to intramedullary haemolysis, homocysteine accumulation due to vitamin B12 deficiency was found to increase haemolysis in vitro. ${ }^{9}{ }^{10}$ pseudothrombotic microangiopathy presents in $2.5 \%$ of patients exhibited In one study of 201 patients with vitamin B12 deficiency. ${ }^{7}$

Its association with Vitamin B12 deficiency has been reported most frequently in adults with pernicious anaemia rather than in those with dietary insufficiency. ${ }^{71}$

Cobalamin is essential co-factor for DNA synthesis and haematopoiesis, and its severe deficiency causes Neutropenia, thrombocytopaenia, \& megaloblastic anaemia, as the case in our patient. ${ }^{12}{ }^{13}{ }^{4}$ (table1)

Our patient had severe pancytopenia and findings of haemolysis (fragmented Red blood cells \& polychromasia) in the peripheral smear mimicking microangiopathic haemolytic anaemia, low reticulocytes in relation to anemia and marked increase in Alkaline phosphatase \& LDH.

Many authors reported a decreased absolute reticulocyte count, and a markedly elevated LDH favor the diagnosis of vitamin B12 deficiency (Psuedo-thrombotic Microangiopathy) over TTP. ${ }^{2}{ }^{71}$

An LDH value exceeding $3000 \mathrm{U} / \mathrm{L}$ strongly favors pernicious anemia. ${ }^{2}$

We found an increase in LDH level of $3707 \mathrm{U} / \mathrm{L}$ in our case.

Furthermore there was dietary and family history of low vitamin D which rises a high suspicion of Nutritional B12 deficiency.

Plasma exchange has no rule in the treatment of B12 deficiency-induced pseudo-TTP..$^{5}$

B12 deficiency, responds dramatically to oral or parenteral vitamin B12.11

Reticulocyte counts reportedly respond to Vitamin B12 administration usually by day five, and the available data suggests that administration of vitamin replacement therapy in such cases is enough without any further intervention. ${ }^{7} 1^{6}$

We achieved a reticulocytes response in our patient together with alkaline phosphatase $\&$ hemoglobin $\&$ decrease in LDH after five days of administration of parenteral Vitamin B12.

However platelet improved by day nine, and we noted a response in absolute Neutrophil Count by day fifteen.

\section{Conclusion:-}

Pseudo- TTP secondary to B12 deficiency should be in the differential diagnosis of patient presenting with microangiopathic hemolytic anemia in childhood.

We highly recommend performing B12 level, homocysteine level, LDH, reticulocyte count \& reviewing peripheral blood smear in suspected TTP cases. 


\section{Acknowledgment:-}

The authors declare no conflict of interest.

\begin{tabular}{|c|c|c|c|}
\hline Age & Gender & Clinical Presentation & Lab \\
\hline $\begin{array}{ll}31 & \text { years } \\
1^{7}\end{array}$ & Male & $\begin{array}{l}\text { SOB, Fatigue and tingling sensation, } \\
\text { inability to cooperate to complete } \\
\text { Neurological Exam. }\end{array}$ & $\begin{array}{l}\text { Hb: } 4.4 \mathrm{~g} / \mathrm{dL} \text { MCV:100.6fL PLT: } 87 \mathrm{~K} / \mathrm{cmm} \\
\text { Total Bilirubin: } 2.7 \mathrm{mg} / \mathrm{dL} \quad \text { Ind. Bilirubin: } \\
1.9 \mathrm{mg} / \mathrm{dL} \\
\text { LDH }>665 \mathrm{U} / \mathrm{L} \text { Haptoglobin: }<7.8 \mathrm{mg} / \mathrm{dL} \\
\text { Normal Coagulation profile } \\
\text { Peripheral Blood Smear: Schistocytes } \\
\text { Cbl: } 68 \mathrm{pg} / \mathrm{mL}\end{array}$ \\
\hline $\begin{array}{l}12 \\
\text { months }{ }^{8}\end{array}$ & Male & Fatigue, bruise $\&$ pallor & 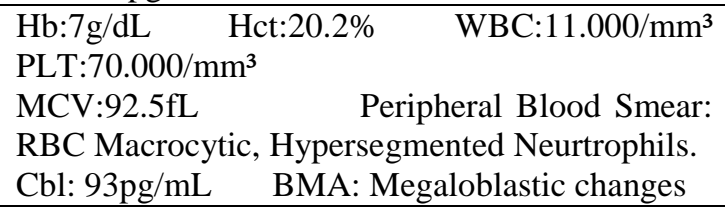 \\
\hline $\begin{array}{l}16 \\
\text { months }{ }^{8}\end{array}$ & Female & $\begin{array}{l}\text { Fatigue, Bruise \& pallor. Received } \\
\text { PRBC transfusion from another center } \\
\text { due to Low amount of RBC. }\end{array}$ & 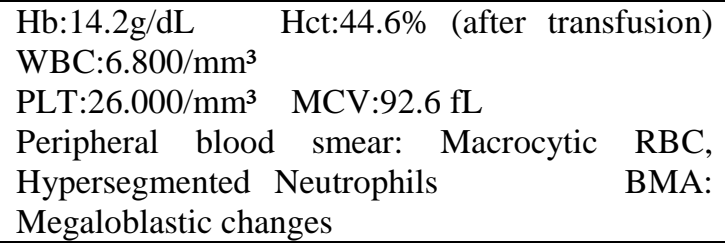 \\
\hline $\begin{array}{l}14 \text { years } \\
16\end{array}$ & Female & $\begin{array}{l}\text { Chronic Abdominal Pain, vomiting } \\
\text { and pallor }\end{array}$ & 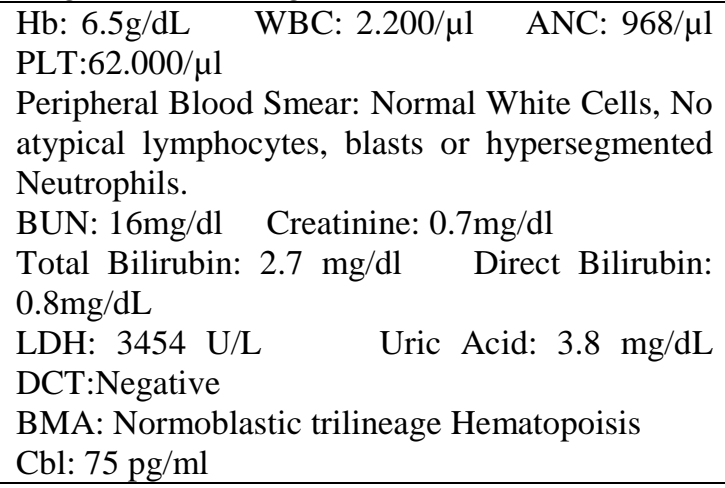 \\
\hline $\begin{array}{l}8 \text { months } \\
19\end{array}$ & Female & $\begin{array}{l}\text { Pallor, Vomiting, Diarrhea \& Failure } \\
\text { to thrive }\end{array}$ & 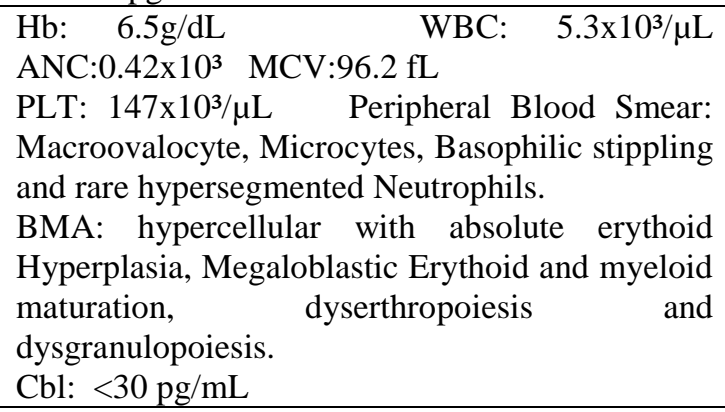 \\
\hline $\begin{array}{l}42 \text { years } \\
\mathbf{2}^{0}\end{array}$ & Female & Fatigue, weakness & 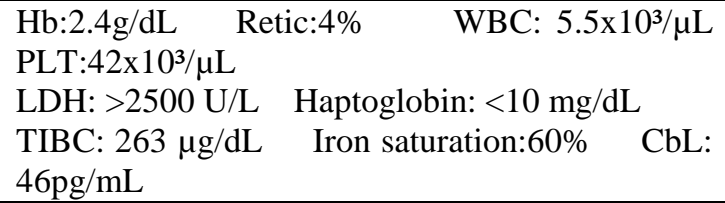 \\
\hline $\begin{array}{ll}42 & \text { years } \\
21 & \end{array}$ & Male & $\begin{array}{l}\text { Weight loss 6-8 pounds over } 2 \\
\text { months, fatigue, presyncope, pale } \\
\text { mucous membrane \& Diabetic } \\
\text { Ketoacidosis. }\end{array}$ & 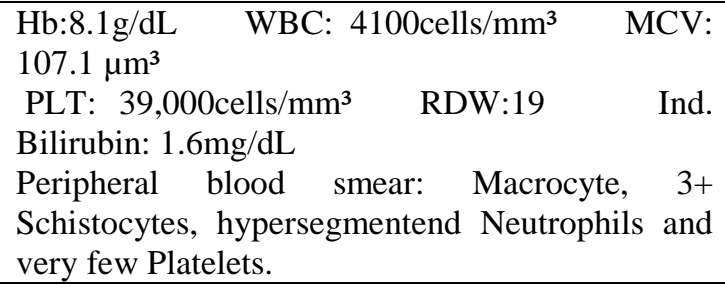 \\
\hline
\end{tabular}




\begin{tabular}{|c|c|c|c|}
\hline & & & $\begin{array}{l}\text { Serum Iron: } 174 \mu \mathrm{g} / \mathrm{dL} \text { TIBC: } 199 \mu \mathrm{g} / \mathrm{dL} \text { iron } \\
\text { Saturation: } 87.4 \% \\
\text { Cbl: } 159 \mathrm{pg} / \mathrm{mL}\end{array}$ \\
\hline 77 years $^{2}$ & Female & $\begin{array}{l}\text { Altered mental status, nausea, } \\
\text { vomiting, bowel incontinence and } \\
\text { bloody diarrhea }\end{array}$ & 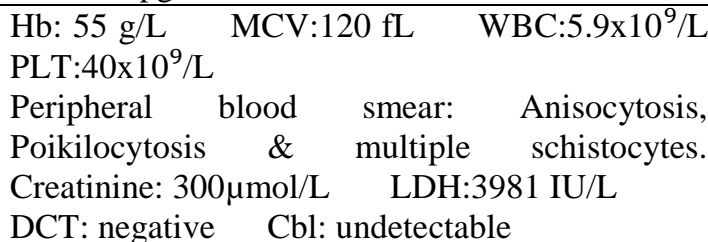 \\
\hline $\begin{array}{l}31 \text { years } \\
22\end{array}$ & Female & $\begin{array}{l}\text { Fatigue, Paresthesia, Scleral icterus } \\
\text { and pallor }\end{array}$ & $\begin{array}{l}\text { Hb: } 5.7 \mathrm{~g} / \mathrm{dL} \text { Hct:15.7\% WBC: } 4,200 / \mathrm{mm}^{3} \\
\text { PLT: } 81,000 / \mathrm{mm}^{3} \\
\text { Total Bilirubin: } 2.2 \mathrm{mg} / \mathrm{dL} \quad \text { Direct Bilirubin: } \\
\text { 0.5mg/dL } \\
\text { LDH: } 4579 \mathrm{U} / \mathrm{l} \\
\text { Numerous schistocytes, anisocytosis, \& Macro- } \\
\begin{array}{l}\text { ovalocytes. } \\
\text { Cbl: } 125 \mathrm{pg} / \mathrm{mL} \quad \text { MMA: } 6258 \mathrm{mmol} / \mathrm{L}\end{array}\end{array}$ \\
\hline $\begin{array}{ll}86 & \text { years } \\
23 & \end{array}$ & Female & $\begin{array}{l}\text { Altered mental status, intubated in ER } \\
\text { due to respiratory distress }\end{array}$ & 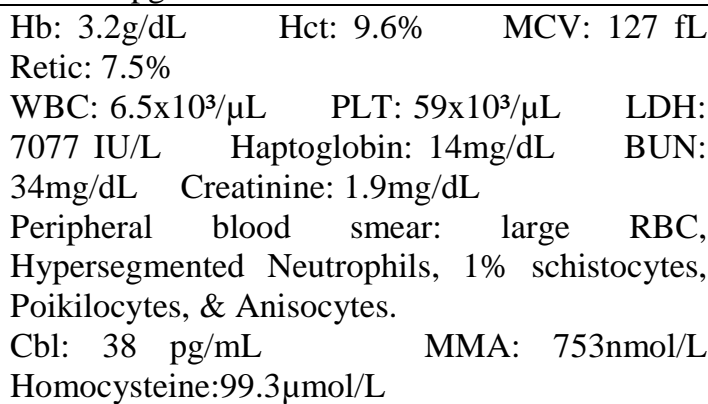 \\
\hline 36 years $^{2^{4}}$ & Male & Fatigue, poor exercise tolerance. & 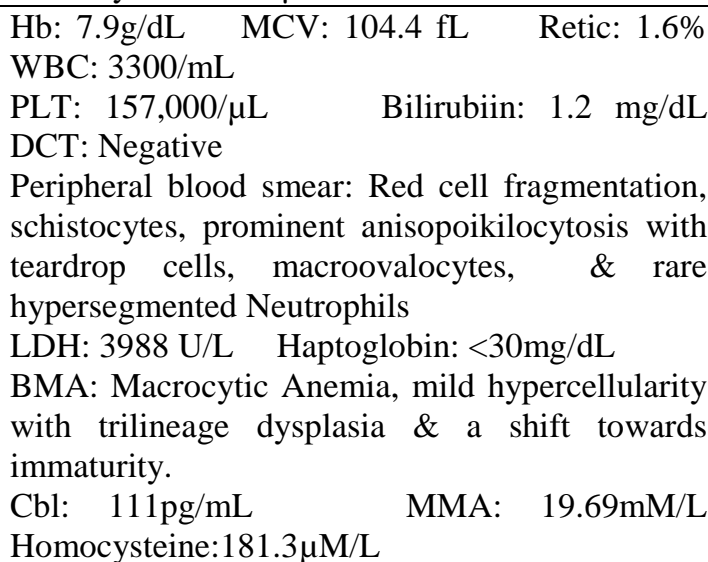 \\
\hline 35 years $^{2^{5}}$ & Male & $\begin{array}{l}\text { Progressive pallor, headache, fever \& } \\
\text { red colored urine. } \\
\text { On physical examination: pale, } \\
\text { jaundice and there was patechia on the } \\
\text { extremities and trunk }\end{array}$ & $\begin{array}{l}\text { Hb: } 5 \text { g/dL Retics: } 30 \% \text { WBC: } 4.4 \times 10^{9} / \mathrm{L} \\
\text { PLT: } 12 \times 10^{9} / \mathrm{L} \\
\text { Peripheral blood smear: Polychromasia, oval \& } \\
\text { crenated cells \& many fragmented cells. } \\
\text { DCT: Negative G6PD: Deficient } \\
\text { LDH: } 2250 \mathrm{U} / \mathrm{L} \\
\text { Normal } \\
\text { BMA: Erythroid hyperplasia. }\end{array}$ \\
\hline $\begin{array}{ll}15 & \text { years } \\
2^{6} & \end{array}$ & Female & $\begin{array}{l}\text { Status Epilepticus, Loss of } \\
\text { consciousness, unable to control } \\
\text { urination. } \\
\text { CT abdomen: slight swelling of the } \\
\text { liver with edema around portal vein. }\end{array}$ & $\begin{array}{l}\text { Hb: } 2.5 \mathrm{~g} / \mathrm{dL} \text { MCV: } 128.8 \mathrm{fL} \text { Retic: } 8.7 \% \\
\text { WBC: } 11600 / \mathrm{mm}^{3} \\
\text { PLT: } 53000 / \mathrm{mm}^{3} \quad \text { Peripheral blood smear: } \\
\text { hypersegmented Neutrophils, red cell Microcytes, } \\
\text { Schistocytes, teardrop cells \& macrocytes. } \\
\text { LDH: } 1903 \mathrm{U} / \mathrm{L}\end{array}$ \\
\hline
\end{tabular}




\begin{tabular}{|c|c|c|c|}
\hline & & & $\begin{array}{l}\text { BMA: Active Hyperplastic trilineage } \\
\text { hematopoiesis with a markedly increased number } \\
\text { of large immature erythroblasts. } \\
\text { Cbl: } 104 \mathrm{pg} / \mathrm{mL} \quad \text { Folic Acid: } 1.4 \mathrm{ng} / \mathrm{mL} \\
\end{array}$ \\
\hline $\begin{array}{ll}15 & \text { years } \\
2^{7} & \end{array}$ & Male & $\begin{array}{l}\text { Vegetarian boy with easy fatigue, } \\
\text { breathlessness, pain on the legs on } \\
\text { walking. } \\
\text { Paleness and icterus in the sclera. } \\
\text { Abdominal U/S: Splenomegaly: } 130 \\
\text { mm in length }\end{array}$ & 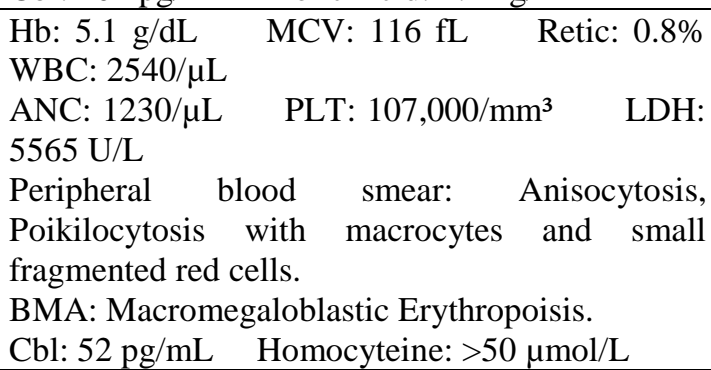 \\
\hline $\begin{array}{ll}33 & \text { years } \\
2^{8} & \end{array}$ & Female & $\begin{array}{l}\text { Woman in } 2^{\text {nd }} \text { pregnancy with } \\
\text { Gestational Age of } 37 \text { weeks and } 3 \\
\text { days. }\end{array}$ & $\begin{array}{l}\text { Hb: } 7.2 \mathrm{~g} / \mathrm{dL} \quad \text { Hct:22.2\% MCV: } 103 \mathrm{fL} \\
\text { WBC: } 9500 / \mu \mathrm{L} \\
\text { PLT: } 42000 / \mu \mathrm{L} \quad \text { Peripheral blood smear: Slight } \\
\text { hypochromia with Macrocytosis }\end{array}$ \\
\hline
\end{tabular}

\section{References:-}

1. Peigne V, Azoulay E, Coquet I, Mariotte E, Darmon M, Legendre P, Adoui N, Marfaing- Koka A, Wolf M, Schlemmer B, Veyradier A. The prognostic value of ADAMTS13 (a disintegrin and metalloprotease with thrombospondin type 1 repeats, member 13) deficiency in septic shock patients involves interleukin-6 and is not dependent on disseminated intravascular coagulation. Crit Care. 2013 Nov 18;17(6):R273.

2. Walter K, Vaughn J, Martin D. Therapeutic dilemma in the management of a patient with the clinical picture of TTP and severe B12 deficiency. BMC Hematol. 2015; 1;15:16.

3. Malla M, Seetharam M. To treat or not to treat: a rare case of pseudo-thrombotic thrombocytopenic purpura in a Jehovah's Witness. Transfusion. 2016;56(1):160-3.

4. McCrae KR, Sadler E, Cines DB. Thrombotic thrombocytopenic purpura and the hemolytic uremic syndrome. In: Hematology: Basic Principles and Practice, 6th Edition. Elsevier: Philadelphia, PA. 1925-1939

5. Stabler SP. Clinical practice. Vitamin B12 deficiency. N Engl J Med 2013;368:149-60.

6. Allen LH. How common is vitamin B-12 deficiency? Am J Clin Nutr 2009;89:693S-6S.

7. Andres E, Affenberger S, Zimmer J, et al. Current hematological findings in cobalamin deficiency. A study of 201 consecutive patients with documented cobalamin deficiency. Clin Lab Haem 2006; 28:50-56.

8. 8- Antony AC. Megaloblastic anemias. In Hoffman R, Benz EJ Jr, Shattil SJ, Furie B, Cohen HJ, Silberstein LE, McGlave P, eds. Hematology, Basic Principles and Practice, 4th ed. Philadelphia, PA: Elsevier, 2005:519556

9. 9- Olinescu R, Kummerow FA, Handler B, et al. The hemolytic activity of homocysteine is increased by the activated polymorphonuclear leukocytes. Biochem Biophys Res Commun 1996;226:912-16.

10. Ventura P, Panini R, Tremosini S, et al. A role for homocysteine increase in haemolysis of megaloblastic anemias due to vitamin B12 and folate deficiency: results from an in vitro experience. Biochim Biophys Acta 2004;1739:33-42.

11. Noël N, Maigné G, Tertian G, et al. Hemolysis and schistocytosis in the emergency department: consider pseudothrombotic microangiopathy related to vitamin B12 deficiency. QJM 2013;106:1017-22.

12. Belen B, Hismi BO, Kocak U. Severe vitamin B12 deficiency with pancytopenia, hepatosplenomegaly and leukoerythroblastosis in two Syrian refugee infants: challenge to differentiate from acute leukaemia. BMJ Case Rep 2014;2014. pii: bcr2014203742.

13. Yenicesu I. Pancytopenia due to vitamin B12 deficiency in a breast fed infant. Pediatr Hematol Oncol 2008;25:365-7

14. Citak FE, Citak EC. Severe vitamin B12 deficiency in a breast fed infant with pancytopenia. J Trop Pediatr 2011;57:69-70.

15. Som S, Deford CC, Kaiser ML, Terrell DR, Kremer Hovinga JA, Lammle B, et al. Decreasing frequency plasma exchange complications in patients treated for thrombotic thrombocytopenic purpura-hemolytic uremic syndrome, 1996 to 2011. Transfusion. 2012;52(12):2525-32.

16. Dimond A, George JN, Hastings C. Severe vitamin B-12 deficiency in a child mimicking thrombotic thrombocytopenia purpura. Pediatr Blood Cancer 2009;52:420-422. 
17. Tuten N, Bennett C, Babcock W. (2015) Thrombotic thrombocytopenic purpura or cobalamin deficiency? A case report and review. Clin Case Rep Rev 1: doi: 10.15761/CCRR.1000152

18. Kömür M, Bayram I, Erbey F, Kücükosmanoğlu. A rare cause of thrombocytopenia in infants: Vitamin B12 deficiency. Eur J Gen Med 2010; 7(1): 107-10

19. Volodymyr Shponka, MD; Maria Proytcheva, MD. Megaloblastic anemia caused by severe B12 deficiency in a breastfed infant. 2017

20. Abbott D. W., Friedman K. D., Karafin M. S. Differentiation of pernicious anemia from thrombotic thrombocytopenic purpura: the clinical value of subtle pathologic findings. Transfusion and Apheresis Science. 2016;55(3):318-322. doi: 10.1016/j.transci.2016.08.005

21. T. Panchabhai, P. Patil, E. Riley, and C. Mitchell, "When the picture is fragmented: vitamin B12 deficiency masquerading as thrombotic thrombocytopenic purpura," International Journal of Critical Illness and Injury Science, vol. 6, no. 2, pp. 89-92, 2016

22. Tadakamalla AK, Talluri SK, Besur S. Pseudo-thrombotic thrombocytopenic purpura: a rare presentation of pernicious anemia. N. Am. J. Med. Sci. 2011; 3 (10): 472-4.

23. Saroj Kandel, Nibash Budhathoki, Shanta Pandey, Bikash Bhattarai, Aam Baqui, Ramesh Pandey, Divya Salhan, Danilo Enriquez, Joseph Quist and Frances M Schmidt. Pseudo-thrombotic thrombocytopenic purpura presenting as multi-organ dysfunction syndrome: A rare complication of pernicious anemia. SAGE Open Medical Case Reports Volume 5: 1-4 2017

24. 24- Veit K. Pseudothrombotic microangiopathy and vitamin B12 deficiency in pernicious anemia. Proceedings (Baylor University Medical Center). 2017;30(3):346-347.

25. 25- Hassan HH, Al-Shareefy LM. The challenge of microangiopathic hemolytic anemia. Iraqi J Hematol 2017;6:24-5

26. 26- Asano, T., Narazaki, H., Kaizu, K., Matsukawa, S., Takema-Tochikubo, Y., Fujii, S., Saitoh, N., Mashiko, K., and Fujino, O. (2015) Neglect-induced pseudo-thrombotic thrombocytopenic purpura due to vitamin B12 deficiency. Pediatrics International, 57: 988-990. doi: 10.1111/ped.12718.

27. Keskin EY, Keskin M. Severe vitamin $B_{12}$ deficiency in a 15-year-old boy: presentation with haemolysis and pancytopenia. BMJ Case Reports. 2015;2015:bcr2015209718. doi:10.1136/bcr-2015-209718.

28. 28- Moghadam, S. and Ghorbani, M. (2016) A Case of Megaloblastic Anemia with Thrombocytopenia. Open Journal of Obstetrics and Gynecology, 6, 534-538. doi: 10.4236/ojog.2016.69068. 